\title{
CT-GUIDED PROCEDURES: AN INITIAL EXPERIENCE
}

\section{ANDREI ROMAN', PATRICIU ACHIMAS-CADARIU ${ }^{2}$, BOGDAN FETICA ${ }^{3}$, VLAD GATA ${ }^{1}$, ANDRADA SEICEAN ${ }^{4}$}

\author{
${ }^{1}$ Radiology Department, "Prof. Dr. Ion Chiricuţă" Oncology Institute , Cluj- \\ Napoca, Romania \\ ${ }^{2}$ Surgery Department, “Prof. Dr. Ion Chiricuță” Oncology Institute , Cluj-Napoca, \\ Romania \\ ${ }^{3}$ Pathology Department, “Prof. Dr. Ion Chiricuță” Oncology Institute, Cluj-Napoca, \\ Romania \\ ${ }^{4}$ Gastrolenterology Department, "Prof Dr Octavian Fodor" Regional Institute of \\ Gastroenterology \& Hepatology, Cluj-Napoca, Romania
}

\begin{abstract}
Background and aims. Despite their usefulness, CT-guided procedures have a low profile in Romania. The current study has the purpose of describing a first experience in performing these procedures.

Methods. Tumors and fluid collections that were inaccessible for biopsy or drainage by ultrasound or endoscopic guidance were included. The procedures were performed using a 64-slice GE Optima CT660 CT scanner. The biopsies were carried out using the coaxial technique with an $18 \mathrm{G}$ semiautomatic needle. The drainages were performed using $10 \mathrm{~F}$ pig-tail drains that were inserted using the Seldinger technique. Data regarding the size and location of the target lesion, puncture technique, success and complication rates were recorded.

Results. Between May 2017 and April 2018, 30 procedures were performed, of which 26 biopsies and 4 drainage insertions. Of the biopsies 3 were mediastinal, 8 pulmonary, 6 retroperitoneal, 4 pelvic, and 5 of the bone. The drainages were performed for pelvic lymphoceles. The average lesion size was $3.2 \mathrm{~cm}(0.7-9 \mathrm{~cm})$, with a depth from the skin of $9.1 \mathrm{~cm}(0.6-15.2 \mathrm{~cm})$. The average procedure duration was 58 minutes (31-93 minutes). A conclusive histopathological diagnosis was set after $92.3 \%$ of biopsies. Three procedures resulted in complications, two being minor (hemothorax, soft tissue hematoma) and one severe (tension pneumothorax requiring drainage).

Conclusions. CT guidance offers safe access to lesions that cannot be biopsied or drained under ultrasound or endoscopic guidance.
\end{abstract}

Keywords: computed tomography, X-Ray, biopsy, drainage, Romania

\section{Background and aims}

The histopathological analysis of tumors has an essential role in their diagnosis and therapy. Tissue samples can be obtained through a variety of methods: open or laparoscopic surgical excision, endoscopic techniques such as gastroscopy, colonoscopy or bronchoscopy, and percutaneous biopsies, the latter of which are performed under imaging guidance. Currently, the most wide-spread guidance technique is ultrasonography (US) because

Manuscript received: 19.08.2018

Received in revised form: 11.09.2018

Accepted: 17.09.2018

Address for correspondence: andrei.roman@umfcluj.ro of its availability, versatility and the lack of exposure to radiation. However, some lesions are not accessible to US-guidance due to their depth or their location within a tissue that is poorly visualized by US such as the lung or bone. A suitable alternative in these cases is offered by CT guidance. This technique, due to an excellent visualization of the anatomical details, allows access to almost any point in the thorax, abdomen, pelvis or limbs.

CT machines had, until recently, a relatively limited spread in Romania, therefore CT guided biopsies have not entered the clinical routine, unless sporadically. The recent wider spread of these machines, as well as the evolution of medicine toward less invasive diagnostic techniques 
have created the opportunity for the development of these procedures. However, the introduction of a new procedure in a medical institution can face some challenges, from assuring the medical personnel and the patients of the benefits and safety of the procedure, to preventing and treating potential complications. The present study has the purpose of describing a prospectively selected group of patients that underwent these procedures while analyzing procedural details, success rates and complications.

\section{Methods}

The present study was approved by the local Ethics Committee. All patients gave informed consent for the interventional procedure and for the administration of iodinated contrast agent when this was deemed necessary.

\section{Patient preparation}

All procedures were performed by a radiologist with one year of experience in CT-guided procedures. The procedure indication was established by the oncologist after consultation with the radiologist. The procedural details, potential complications and diagnostic alternatives were

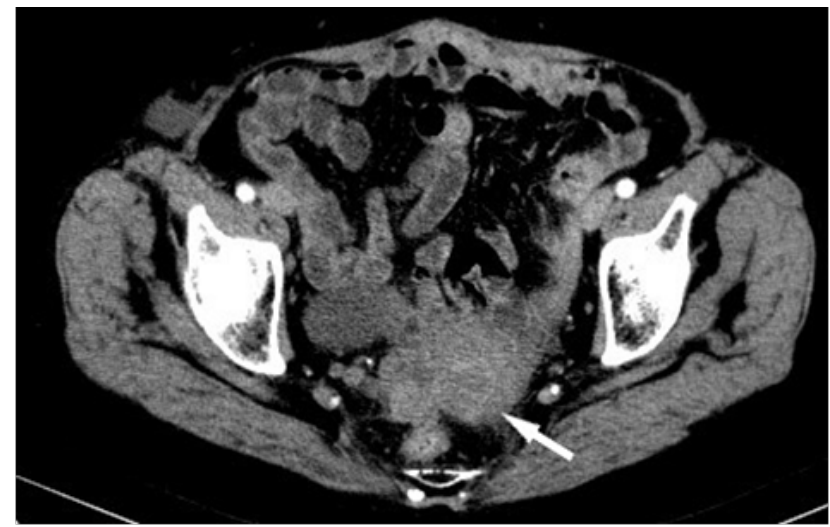

$\mathbf{a}$

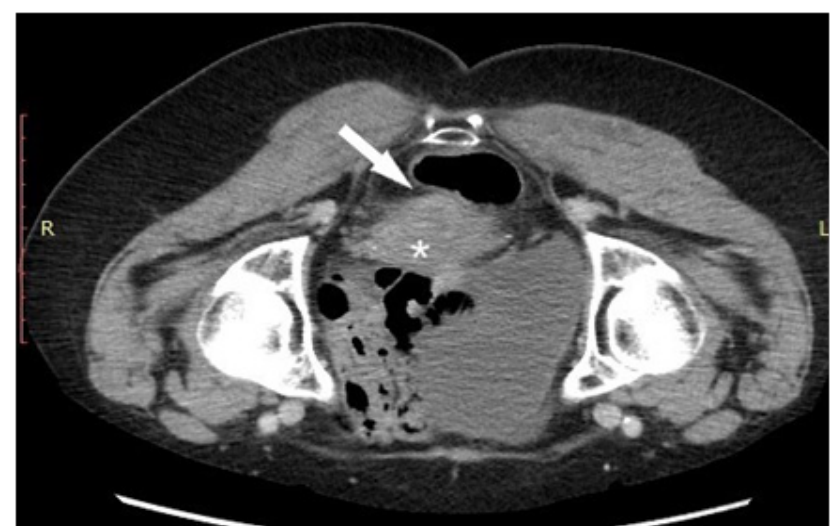

c

Figure 1. A 76-year-old female patient known with a previously resected colorectal carcinoma. a) A follow-up CT showed two enhancing suspicious anteriorly located pararectal lesions (arrow). b) An MRI performed shortly thereafter confirmed the presence of the lesions, as well as their malignant appearance. On the DWI sequence, the lesions show diffusion restriction which is suggestive for malignancy. c) The contrast enhanced planning CT, with the patient in prone position, allows the visualization of the left tumor (arrow), located between the rectum posteriorly, and the uterus anteriorly (asterisk). Due to the prone position and to the air in the rectum the anatomical relationships are changed compared to the previous exams. d) The tumor was reached using an infrapiriform parasacral approach. Due to the homogeneous contrast enhancement in the late phase, the tumor cannot be directly visualized. For this reason, the biopsy was performed based only on topographical criteria. Some perirectal air (arrow) can be seen that entered through the coaxial needle. There was no suspicion of rectum perforation. explained to the patient. Previous imaging exams were evaluated in order to establish if the lesion was accessible for puncture. Similarly, all patients, except those with pulmonary or bone lesions were examined using US in order to exclude the possibility of US guidance. The coagulation status, including APTT, INR and thrombocyte count, as well as the creatinine clearance were verified prior to the intervention. Anticoagulant and antiplatelet therapy were ceased according to the coagulation management guidelines for percutaneous image-guided interventions [1].

\section{Biopsy technique}

All procedures were performed using the same CT machine (GE Optima CT660, 64 slices; General Electric, USA, $5 \mathrm{~mm}$ collimation). For soft tissue biopsies (Figure 1), a coaxial biopsy set (Somatex Medical Technologies, Berlin, Germany) was used, which included a $17 \mathrm{G}$ coaxial needle $(5 / 10 / 15 \mathrm{~cm}$ long) and the corresponding $18 \mathrm{G}$ semiautomatic biopsy needle $(10 / 15 / 20 \mathrm{~cm})$. The length of the coaxial and biopsy needles was chosen depending on the depth of the lesion. Bone biopsies were performed using an $11 \mathrm{G}$ bone marrow biopsy needle (Figure 2).

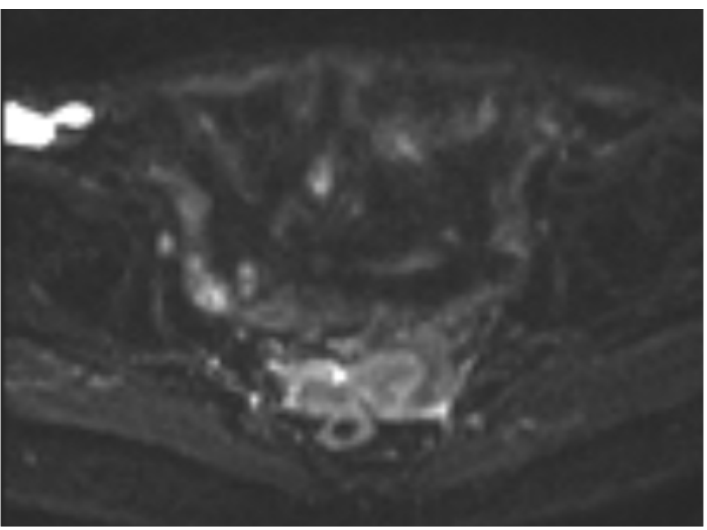

b

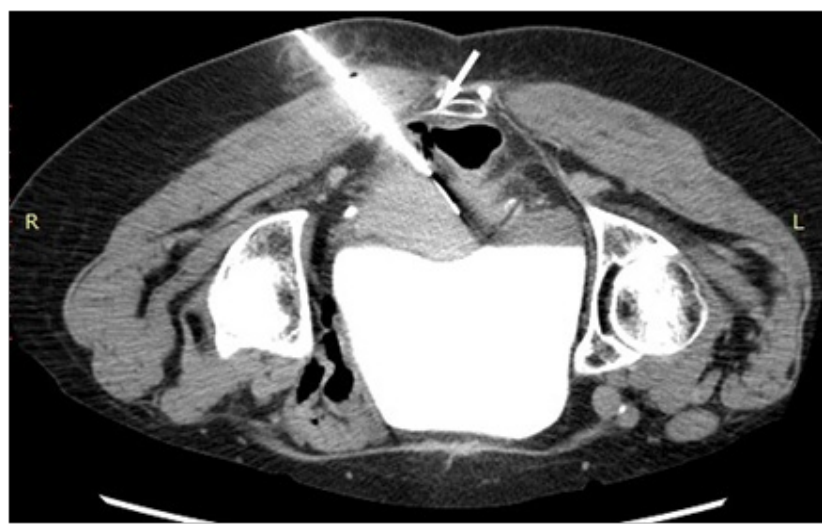

d 

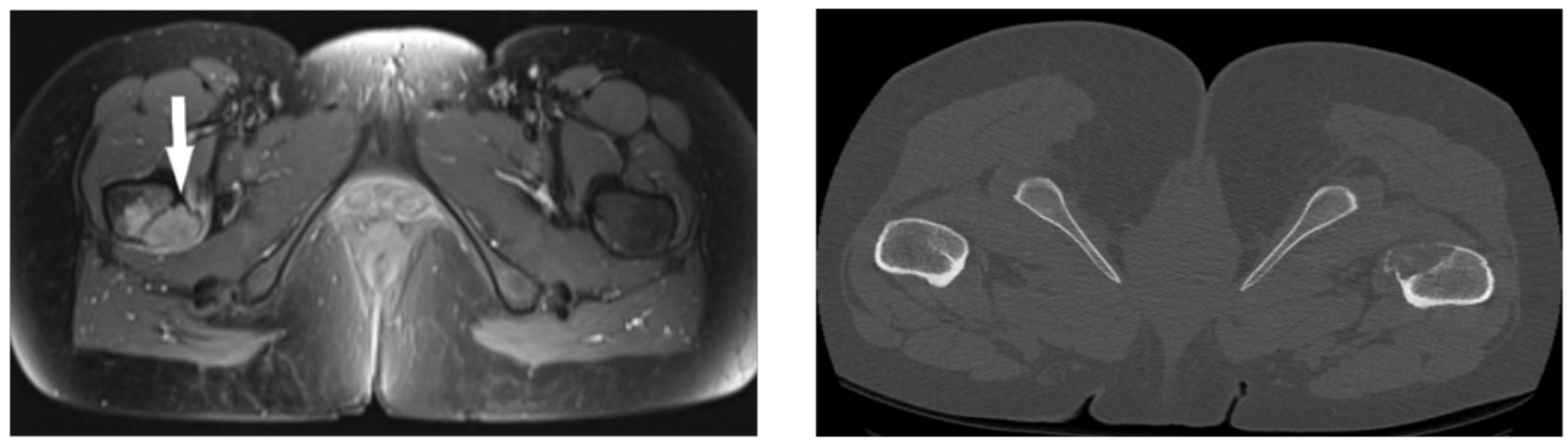

a

b

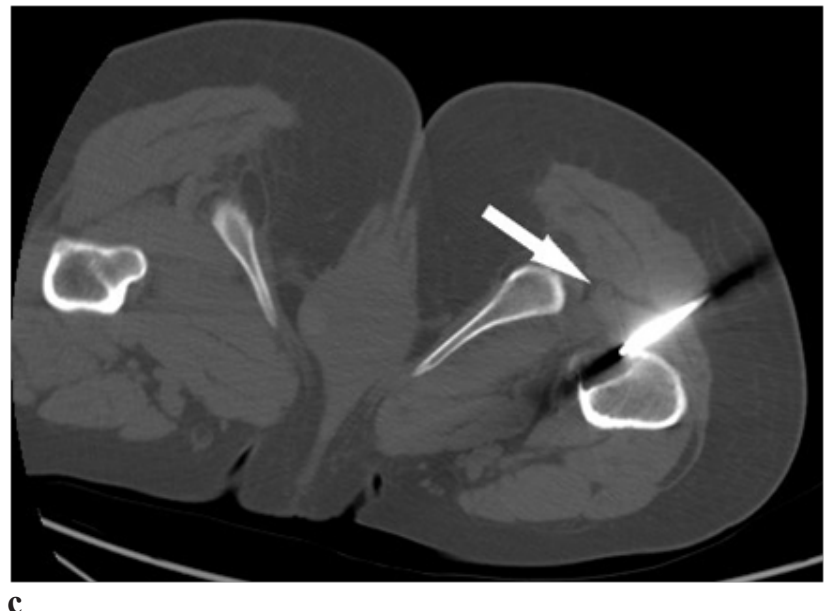

Figure 2. A 43-year-old female patient, with a newly diagnosed breast cancer had performed a staging CT (not shown) on which a single suspicious osteolytic lesion was found on the lesser right trochanter. a) A contrast-enhanced MRI exam shows significant enhancement of the lesion (arrow). b) The planning CT in prone position shows the marked osteolytic lesion with a thin osseous rim at the periphery. c) The biopsy was performed using an oblique lateral approach in order to avoid the sciatic nerve (arrow) and so that long axis of the tumor would be accessible. The needle tip can be seen immediately after it had pierced the thin osseous rim.

The patient did not eat or drink on the day of the procedure. An unenhanced planning CT was performed immediately prior to the procedure. Iodinated contrast agent was administered if lesion visualization was unsatisfactory or if vital anatomical structures such as large blood vessels were in close proximity.

The safest puncture path was chosen based on the planning CT. Metallic markings were placed on the skin during the planning $\mathrm{CT}$ for proper orientation. The slice selected for the biopsy was identified using the gantry`s laser, and the exact puncture spot was chosen on the skin using the metallic markers. The procedure was performed under sterile conditions. The skin and the peritoneum/ pleura were locally anesthetized using xylocaine. The coaxial puncture needle was introduced by successive repositionings under $\mathrm{CT}$ guidance with single-slice scans or series of three scans if the needle exceed the initial plane. After the tip the coaxial needle reached the edge of the lesion, the biopsy needle was inserted using its outer cannula as an introducer. In most cases, three tissue samples were considered sufficient. From high-risk locations, such as the lung, or perivascular tumors, or in the case of hypervascular tumors where significant blood extravasation through the coaxial needle was noted only 1-2 samples were obtained. The tissue samples were stored in formalin and delivered to the pathology department for analysis. Signs of complications such as bleeding or pneumothorax were continuously looked after during the procedures. The procedures were finalized if a control scan performed 5 minutes after the removal of the coaxial needle showed no complications.

\section{Drainage technique}

Drainages were used for the treatment of pelvic lymphoceles. The lymphoceles occurred as a consequence of lymph drainage impairment in patients who underwent pelvic lymphadenectomy during gyne-oncologic surgery. Only symptomatic lymphoceles due to deep vein thrombosis or persistent pain were treated. In order to avoid the risk of recurrence consecutive to a simple aspiration, a drain tube was inserted in all cases.

The procedures were performed using a percutaneous drainage set (Somatex Medical Technologies, Berlin, Germany) that included a $10 \mathrm{~F}$ pig-tail catheter (Figure 3 ). The drainage procedure was identical to that 


\section{Medical Imaging}

of the biopsies until the insertion of the coaxial needle. For the confirmation of the correct needle placement, a few milliliters of fluid were extracted using a syringe. The guidewire was, then, inserted in the lymphoceles through the coaxial needle. After the acquiring a new image to confirm the position of the guidewire, the coaxial needle was removed and the puncture tract was enlarged with the dilators included in the drainage set. The drainage was finally introduced in the lymphoceles and the guidewire removed. The drainage was secured to the skin with a surgical wire and the fluid was completely drained before performing the final control scan. The tube was removed when the daily drainage volume dropped under $25 \mathrm{ml}$.

\section{Post-procedural follow-up}

During the first two hours following the procedure the patient's vital signs were closely monitored. In the case of pulmonary or mediastinal procedures, a chest radiograph was performed after two hours in order to exclude a pneumothorax or a hemothorax. If no complications occurred, the patient was released on the following day after being given instructions regarding the unlikely possibility of delayed complications.

\section{Study parameters}

For the purpose of the present study, following procedural parameters were recorded: patient age and gender, lesion size, location and depth, the distance to a vital anatomical structure, the number of tissue samples obtained and the final pathological diagnosis. Other parameters related to the scanning protocol were the usage of contrast agent, patient position, number of scans, procedure duration (from the time of the planning scan to the last control scan). The total radiation dose (the planning $\mathrm{CT}$ and the guidance scans) and the procedure dose (only the guidance scans) were recorded using the dose-length product measured in $\mathrm{mGy}^{*} \mathrm{~cm}$. The complications were classified as minor or major, the latter requiring additional therapeutic measures.

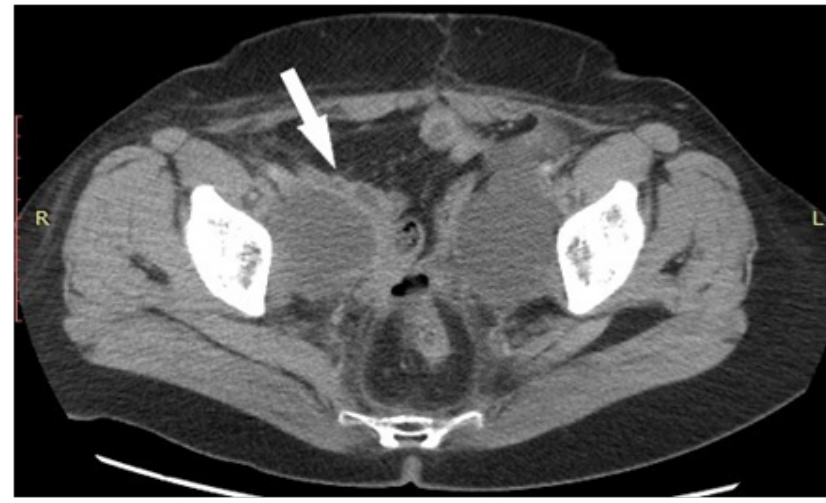

a

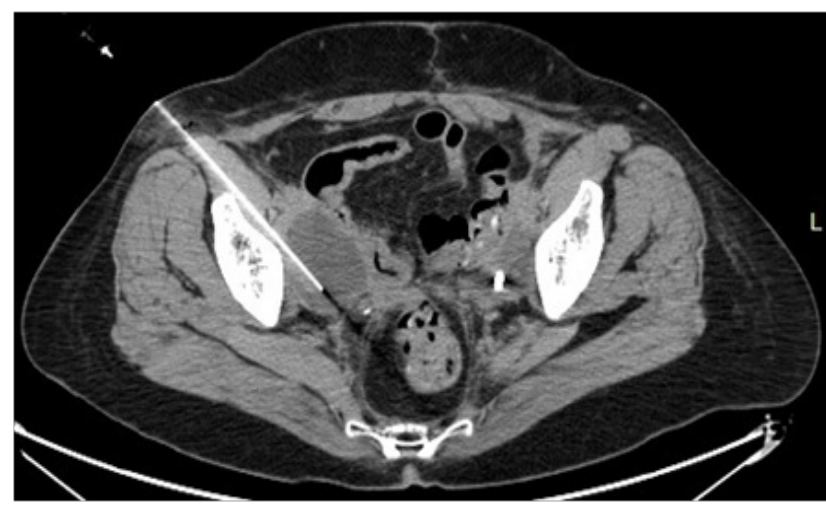

c

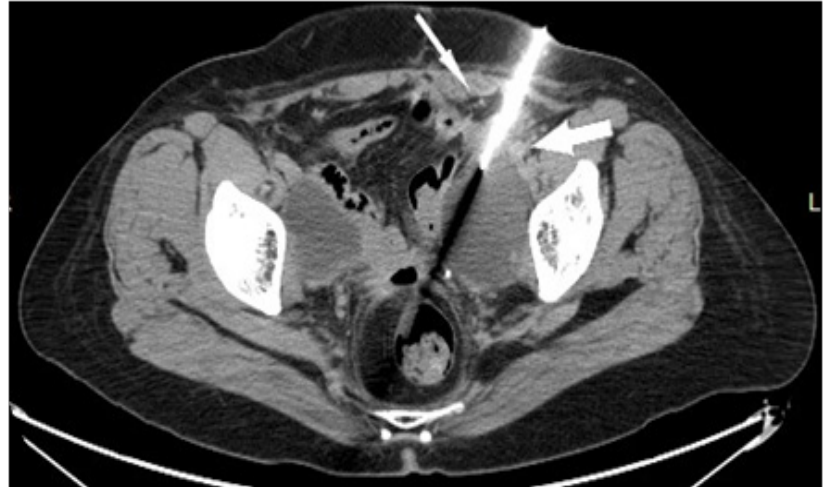

b

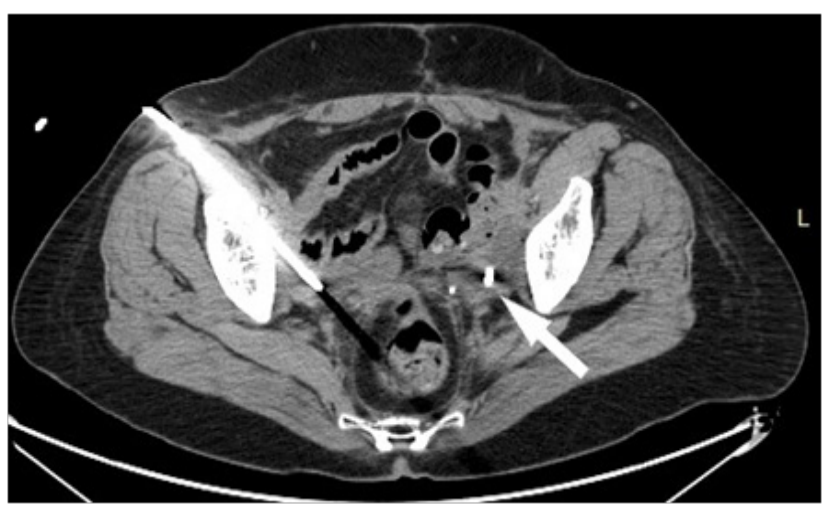

d

Figure 3. A 50 years old woman with a resected cervical cancer and bilateral lymphadenectomy had a pelvic US exam due to a left deep vein thrombosis and pelvic pain. The US exam identified two deeply located pelvic lymphoceles. a) The planning CT shows the two large lymphoceles that compress the external iliac veins. On the right side, small bowel loops (arrow) can be seen anteriorly to the lymphoceles, excluding the possibility of an anterior approach. b) Drainage of the left lymphoceles. The small window available between the external iliac vessels (thick arrow) and the inferior epigastric vessels (thin arrow) required CT guidance. c) Drainage of the right lymphoceles. The drainage was inserted through the iliopsoas muscle in order to avoid damage to the anterior bowel loops. d) Both tubes have been inserted and the lymphoceles are completely drained. The right drain can be visualized along its full path, whereas on the left side only the distal loop of pig-tail catheter can be seen (arrow). 
Table I. Procedure characteristics classified after body region

\begin{tabular}{|c|c|c|c|c|c|c|c|c|c|c|c|}
\hline Body region & $\begin{array}{l}\text { Procedures } \\
\mathrm{n}\end{array}$ & $\begin{array}{l}\text { Lesion } \\
\text { size } \\
(\mathrm{cm}) \\
\end{array}$ & $\begin{array}{l}\text { Lesion } \\
\text { depth } \\
(\mathrm{cm})\end{array}$ & $\begin{array}{l}\text { Scans } \\
\mathrm{n}\end{array}$ & $\begin{array}{l}\text { Total dose } \\
\left(\mathrm{mGy}{ }^{*} \mathrm{~cm}\right)\end{array}$ & $\begin{array}{l}\text { Guidance } \\
\text { dose } \\
(\mathrm{mGy} * \mathrm{~cm})\end{array}$ & $\begin{array}{l}\text { Duration } \\
\text { (min) }\end{array}$ & $\begin{array}{l}\text { Samples } \\
\mathrm{n}\end{array}$ & $\begin{array}{l}\text { Diagnostic } \\
\text { success } \\
\mathrm{n}(\%) \\
\end{array}$ & $\begin{array}{l}\text { Minor } \\
\text { complications } \\
\mathrm{n}(\%)\end{array}$ & $\begin{array}{l}\text { Major } \\
\text { Complications } \\
\mathrm{n}(\%)\end{array}$ \\
\hline Lung & 8 & 2.5 & 9.1 & 26.8 & 650.2 & 299.7 & 49.8 & 2.3 & $8(100 \%)$ & $1(12.5 \%)$ & $1(12.5 \%)$ \\
\hline Mediastinum & 3 & 5.4 & 8.7 & 23.7 & 1320.3 & 229.3 & 53.7 & 2.3 & $3(100 \%)$ & $1(33.3 \%)$ & 0 \\
\hline Retroperitoneum & 6 & 4.4 & 12.5 & 22.7 & 1360.2 & 249.2 & 58.8 & 2.7 & $5(83.3 \%)$ & 0 & 0 \\
\hline Pelvis & 4 & 2.7 & 6.9 & 25.7 & 1096.0 & 375.2 & 59.7 & 3.0 & $4(100 \%)$ & 0 & 0 \\
\hline Bone & 5 & 1.6 & 8.6 & 29.6 & 1092.6 & 616.8 & 73.2 & 2.2 & $4(80 \%)$ & 0 & 0 \\
\hline Drain & 4 & 3.6 & 7.1 & 17.2 & 714.2 & 234.2 & 56.0 & $\mathrm{~N} / \mathrm{A}$ & $\mathrm{N} / \mathrm{A}$ & 0 & 0 \\
\hline
\end{tabular}

\section{Results}

Between 30.05.2017-02.04.2018, 30 CT-guided procedures (26 biopsies and 4 drainage placements) have been performed on 29 patients, of which 12 were female and 17 male. The patients' average age was 52.8 years old, ranging between 18-81 years old. Biopsies were taken from $3(11.5 \%)$ mediastinal, $8(30.7 \%)$ pulmonary, $6(23.1 \%)$ retroperitoneal, 4 (15.4\%) pelvic, and 5 (19.2\%) bone tumors. All drainages were performed on pelvic lymphoceles (Table I).

The planning exams included in 20 cases $(66.6 \%)$ a single sequence, of which 15 unenhanced, 2 in the arterial phase and 3 in the venous phase, in 3 cases $(10 \%)$ two sequences (unenhanced and venous phase) and in 7 cases $(23.3 \%)$ three sequences (unenhanced, arterial and venous). Half of the planning exams were performed after the administration of contrast agent. Thirteen (43.3\%) procedures were performed in supine position, while 17 $(56.6 \%)$ were performed in prone position.

The average size of the target lesions was 3.2 $(0.7-9) \mathrm{cm}$, with a depth of $9.1 \mathrm{~cm}(0.6-15.2) \mathrm{cm}$. In 15 cases, vital structures were located in close proximity to the puncture tract with an average distance of $0.6(0.1-1.2)$ $\mathrm{cm}$. On average, 2.5 tissue samples were collected, a single sample being collected in $3(11.5 \%)$ cases, two samples in 7 $(26.9 \%)$ cases and 3 samples in $16(61.5 \%)$ cases.

The average procedure duration was 58.0 (31-93) minutes. For the needle guidance, 19.4 (11-42) single slice scans/procedure and $5.5(0-14)$ triple scans/procedure were used. The total radiation dose, which included both the planning CT and the guidance scans was 1019.0 (384.02600.0) $\mathrm{mGy}^{*} \mathrm{~cm}$, while the guidance scans-only dose was 336.2 (138.0- 835.0) $\mathrm{mGy} * \mathrm{~cm}$.

For 24 patients $(92.3 \%)$, the biopsy offered enough material for a definitive histopathological diagnosis. In two situations, the biopsy was non-contributive. The first case was of a patient with retroperitoneal lymph nodes suspected of lymphoma. Although the lymph node was successfully reached with the biopsy needle, no satisfactory tissue samples could be extracted. The second case was of an osseous biopsy from a PET/CT-positive tumor within the femur shaft. Only a small sample could be extracted which was subsequently contaminated during the fixation process, becoming uninterpretable.
Three procedures (10\%), all of them involving the thorax, led to complications. Two complications were minor, one being a self-limited hemothorax that occurred during the biopsy of a mediastinal solitary fibrous tumor, while the other was a soft tissue hematoma that occurred because of a lesion to the intercostal vessels during the biopsy of a pulmonary tumor. The major complication was a tension pneumothorax that occurred after the biopsy of a lung tumor in a patient with emphysema (Figure 4). The pneumothorax was treated with the insertion of a chest tube that was maintained for two days. No late complications or procedure-related deaths were encountered.

The drains included in the present study were inserted and maintained without complications. No sclerosing agents were used and the longest drainage period was 4 weeks.

\section{Discussion}

CT-guided procedures emerged and developed in parallel with CT technology, the first CT-guided biopsy being performed in 1975 on a retroperitoneal tumor, just four years after CT was introduced in the clinical practice [2]. Shortly thereafter, these procedures had become widespread, their usage gaining momentum in the 90 `s after the introduction of multi-slice spiral CT technology[3].

CT-guided procedure are currently part of the diagnostic routine in many medical centers, in some of which, due to the large number of interventions, there are CT machines dedicated to this purpose. All CT manufacturers offer hardware and software extensions for CT-guided procedures with the purpose of improving the working condition, thus reducing the radiation doses and procedure duration. The hardware components consist of monitors, a joystick and radiation protection screens located at the CT table. The software extension facilitates procedure planning through advanced image reconstruction and three-dimensional orientation options, and allow for real-time comparison between the planning images and the guidance scans [4].

Using these extensions, the physician can work safely and fast, without having to leave the CT room for each scan in order to view the images and to avoid being irradiated. The absence of these instruments, as well as the initial lack of experience of the technologists led to some 
difficulty in performing the procedures which was reflected in the high number of scans (over 19 per procedure), the need to use triple scans, both increasing the overall radiation dose and procedure duration which lasted, on average, for an hour. The lack of a command joystick and in-room monitors make pulmonary interventions particularly difficult because these often require both image acquisition and needle manipulation to be made during the same breath hold. Despite these limitations, the lesions were successfully targeted in all cases.

For percutaneous interventions, the guidance method can be chosen from a wide spectrum of techniques. Lesions concerning the digestive tract and the tracheobronchial tree are almost exclusively biopsied using endoscopic procedures. Deep mediastinal tumors and large pulmonary tumors located close to large bronchi are more readily accessible for endobronchial ultrasound (EBUS) guided biopsies $[5,6]$. The possibility of ultrasound guidance should be excluded first for all other tumors or fluid collections, irrespective of their location. US is widely available, does not use ionizing radiation and does not interrupt the workflow of a CT machine. This procedure is most well-suited for superficial abdominal and pelvic lesions as well as for pulmonary lesions that are in direct contact with the pleura. US-guidance is limited by the acoustic attenuation encountered in the case of deep tumors and obese patients which often impedes satisfactory lesion and needle visualization. Likewise air-containing structures and bones are not penetrated by ultrasound waves, making structures located within or behind them inaccessible via US guidance. In the majority of these situations, CT guidance will provide the means for biopsy or drainage [7-9]. Other guidance techniques such as fluoroscopy and MRI have been reported. Fluoroscopic guidance was used in the past for the biopsy of pulmonary tumors, but has been completely replaced by CT guidance due to its vastly superior accuracy and safety. MRI guidance is currently required for some very specific indications such as breast tumors that cannot be visualized using US or mammography as well as for prostate biopsies.

Most of the patients included in the present study were referred for CT guided biopsy after the lesions were considered inaccessible for US-guided or following inconclusive bronchoscopic exams in the case of lung tumors. For all these patients, the only alternative for a histopathological diagnosis would have been a surgical biopsy.

Percutaneous biopsies can be performed using either the fine needle aspiration (FNA) technique or core needle $(\mathrm{CN})$ sampling. Using FNA, only individual cells can be sampled, with no information regarding to tissue architecture, but immunohistochemical and molecular analysis is still possible. The advantage of FNA consists in the use of thinner, less traumatic needles that do not require an excursion motion for sampling, but at the cost of less material and no histological information. Consequently, FNA is to be preferred in the case of small lesions, located close to vital structures, such as lymph nodes or the thyroid [10].

$\mathrm{CN}$ systems are based on needles with a small notch at their tip that is quickly covered by a cylindrical outer sheath upon activation, thus capturing the tissue sample. The diameter of these needles vary between $20-14 \mathrm{G}$ with a notch length of $12-22 \mathrm{~mm}$. The activation can be automatic whereby the needle tip and the outer sheath are mechanically launched in a short succession, or semiautomatic whereby the needle tip is manually inserted in the tumor and only the outer sheath is mechanically launched. The advantage of $\mathrm{CN}$ biopsies is the ability to obtain larger samples with preserved histological structure, but they require the use of thicker needles. A systematic literature review reported diagnostic accuracies between $79.7-91.8 \%$ for FNA and $89.0-96.9 \%$ for $\mathrm{CN}$, respectively, but the data were not sufficient to be able to state the superiority of one technique over the other [11]. Considering the lack of consensus over the optimal technique, the choice belongs to the performing physician, while taking the experience of the pathologist in reading histological and cytological samples into account. At our institution, we perform $\mathrm{CN}$ biopsies using the coaxial technique which allows obtaining more tissue samples, without the need of multiple passes. Thus, with a success rate of $92.3 \%$, our results are comparable with those reported in the aforementioned review. For CT-guided biopsies we use exclusively semiautomatic devices because they allow the controlled insertion of the notched needle tip and because of their small handle that easily fits inside the gantry. The light weight of the handle is also important because it reduces the risk of needle displacement when it is not manually sustained during scans.

The biopsy of pulmonary tumors is one of the most frequent CT-guided procedures. According to a recent prospective randomized study that compared the diagnostic accuracy between CT-guided and EBUS-guided biopsies the results were significantly in favor of CT guidance with rates of $85 \%$ and $65 \%$, respectively[5]. In the case of centrally located, large tumors, the accuracy of EBUS nears that of $\mathrm{CT}$, being a viable alternative, especially because of its lower risk of pneumothorax. CT guidance, however, offers significantly greater precision for smaller, more peripheral located tumors $[5,12]$. Performed by an experienced radiologist, biopsies of very small tumors can have satisfactory success rates as shown by Chang et al who reported a diagnostic accuracy of $87.1 \%$ for tumors $<8 \mathrm{~mm}$ [12]. In our patient series the success rate for lung biopsies was $100 \%$, but the small number of patients and the relatively large tumor size should be taken into consideration.

High success rates have been reported for CT-guided biopsies of other organs: liver, $86-100 \%[8,13]$; retroperitoneal tumors, $95.9 \%$ [14]; pancreas, $>90 \%$ [15,16]; adrenal glands, $96 \%$ [17]; kidney, 79-90.8\% [18,19]; bone, >94\% [20,21]. With an overall biopsy success rate of $92.3 \%$, our results were similar with those reported in the literature. 
The complications related to CT-guided procedures are hemorrhage (hematomas, hemoperitoneum, hemothorax, lung hemorrhage), pneumothorax, infection caused by digestive tract perforation, nerve lesions, arterio-venous fistulas after hepatic and renal biopsies, pathological bone fractures and needle tract seeding. Among the different types of CT-guided biopsies, the pulmonary procedures have the highest complication rates. According to a recent meta-analysis, the risk of pneumothorax after $\mathrm{CN}$ lung biopsy is $25.3 \%$, of pneumothorax requiring the insertion of a chest tube is $5.6 \%$, of lung hemorrhage is $18.0 \%$ and of lung hemorrhage with hemoptysis is $4.1 \%$ [22].

The three complications that we encountered occurred as a consequence of thoracic biopsies. In the case of the first patient, during the biopsy of a hypervascular mediastinal tumor (solitary fibrous tumor), the buildup of a hemothorax could be noticed, which at the end of the procedure was $2 \mathrm{~cm}$ thick. Control scans were performed at 5, 10 and 15 minutes and the hemothorax showed no further signs of progression. The patient was closely monitored in the following hours, and was released the next day.

The second patient had a biopsy of a lung tumor

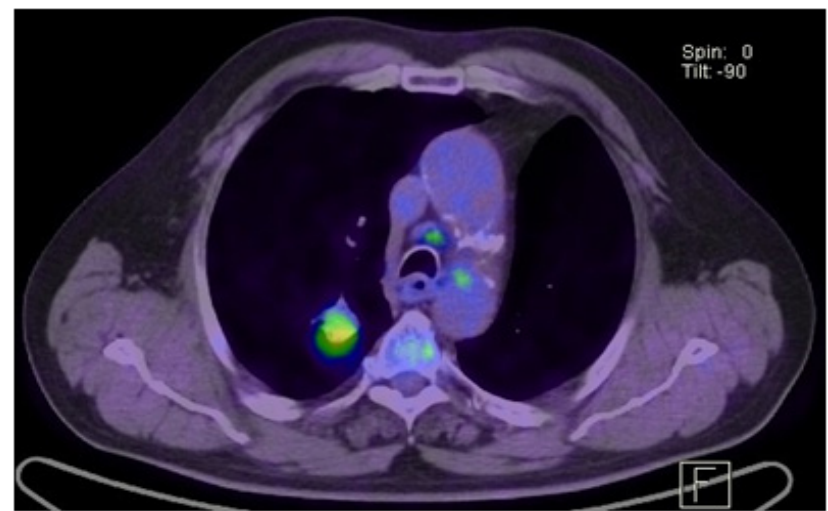

a

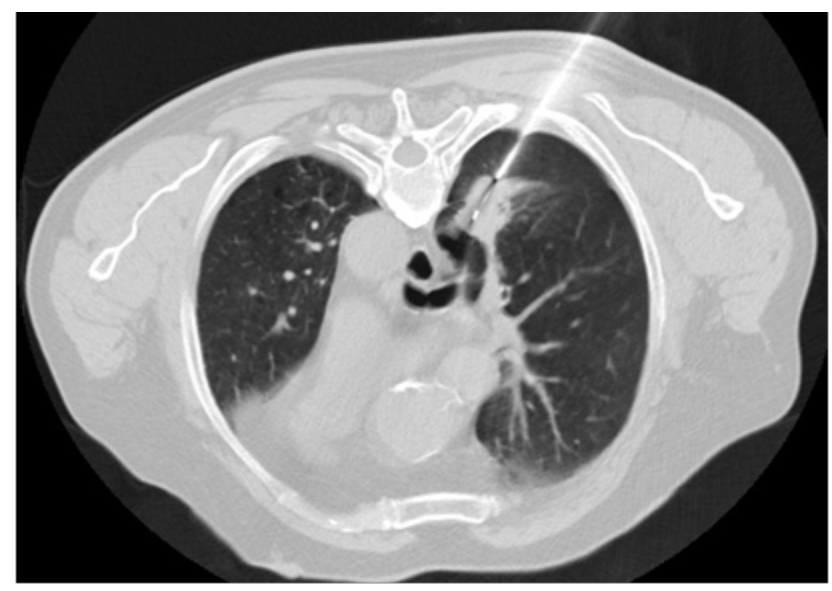

c

Figure 4. A 47 years old patient, with a previously operated lower left lobe large cell carcinoma had a follow-up CT on which a new upper right lobe tumor could be found. a) The subsequent PET/CT exam shows that the tumor is metabolically active, and thus most likely malignant. b) The planning CT (on a slice cranially to the tumor) shows extended areas of centrilobular emphysema (arrows), thus increasing the likelihood of a pneumothorax. c) The biopsy was performed using a posterior oblique approach. The upper segment of the lower right lobe and the oblique fissure were traversed by the needle. No signs of pneumothorax could be noticed at this point. d) Radiography performed two hours after the procedure showing a tension pneumothorax. located in the right upper lobe (scuamous carcinoma). Due to the patient's lack of cooperation leading to ample respiratory motions, multiple needle repositionings were required, which led to the injury of the intercostal vessels. As a consequence, a $4 \mathrm{~cm}$ thoracic wall hematoma formed and was treated conservatively.

The third patient (Figure 4) also had a biopsy of a tumor located in the right upper lobe (adenocarcinoma). The patient was known with centrilobular emphysema, and due to the high pneumothorax risk, the surgeon and anesthetist were noticed about the procedure in advance. Immediately after the needle was removed, a small pneumothorax could be noticed, which was stable at a scan performed $10 \mathrm{~min}$ after. The patient was transferred to the ward, where he was closely monitored. Two hours after the procedure, the patient had dyspnea, was cyanotic and had a blood oxygen saturation of $85 \%$. A chest radiograph was immediately performed and showed a tension pneumothorax with a width of $5 \mathrm{~cm}$. A chest tube was subsequently inserted and the symptomatology subsided immediately. The chest tube was kept for two days and the patient was released the day after its removal.

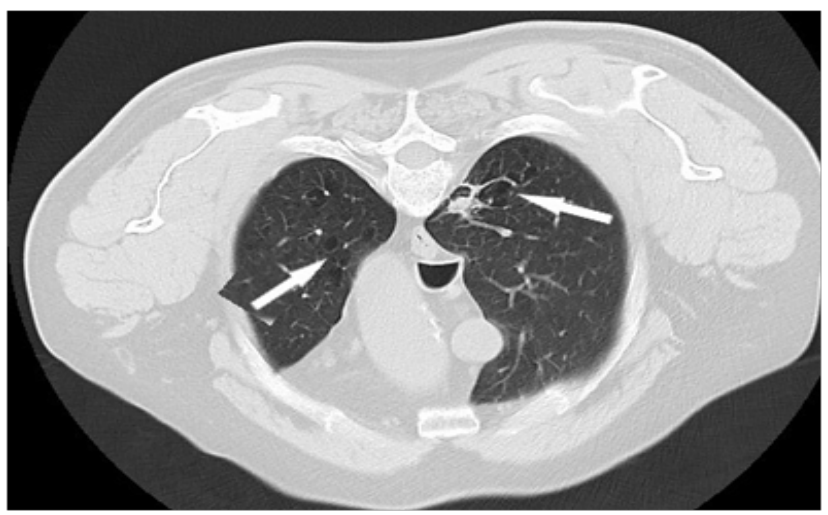

b

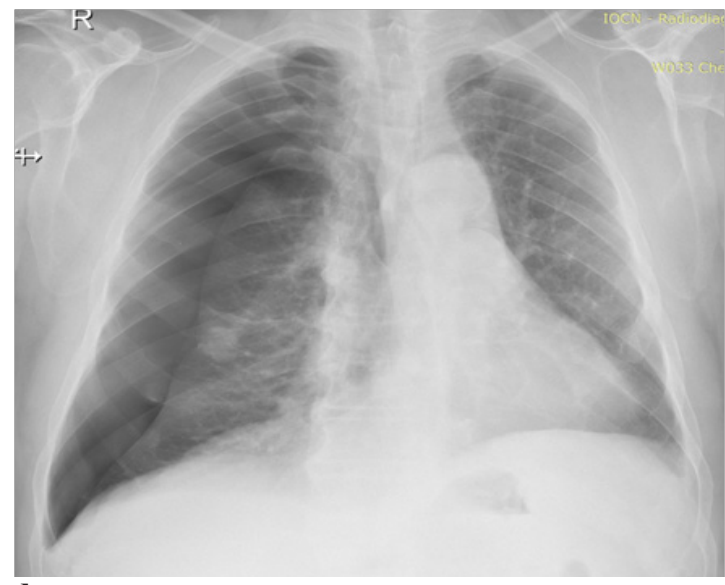

d 
The insertion of a percutaneous drainage is an efficient minimally-invasive treatment option for fluid collections. Lymphoceles are fluid collections that occur as a consequence of the impairment of lymphatic drainage after pelvic lymphadenectomy or renal transplantation. Although they usually are asymptomatic and do not require treatment, lymphoceles can cause pain or deep vein thrombosis through compression of nerves or veins or can become infected, in which case they have to be drained. The insertion of a drainage tube is usually necessary because lymphoceles have a high recurrence rate. The tube should stay in place until the drainage volume drops significantly, which can last for a few weeks. In order to shorten this period, sclerosing agents such as ethanol or bleomycin can be injected over the drain [23]. In our study, all lymphoceles were successfully drained, without complications or the need for sclerosing agents.

\section{Conclusions}

CT-guided interventional procedures represent a safe and effective modality for obtaining biopsies from tumors and draining fluid collections that are inaccessible by means of endoscopic or US guidance. Despite the difficulties encountered as a result of the lack of a dedicated CT machine and the fact that the interventions were performed in an institution with no previous experience involving interventional radiological procedures, the success and complication rates have been comparable with those previously reported in the literature.

\section{References}

1. Patel IJ, Davidson JC, Nikolic B, Salazar GM, Schwartzberg MS, Walker TG, et al. Consensus guidelines for periprocedural management of coagulation status and hemostasis risk in percutaneous image-guided interventions. J Vasc Interv Radiol. 2012;23:727-736.

2. Alfidi RJ, Haaga J, Meaney TF, MacIntyre WJ, Gonzalez L, Tarar R, et al. Computed tomography of the thorax and abdomen; a preliminary report. Radiology. 1975;117:257-264.

3. Cerci JJ, Bogoni M, Delbeke D. History of Image-Guided Biopsy. Oncological PET/CT with Histological Confirmation: Springer; 2016. pp 1-7.

4. Faiella E, Frauenfelder G, Santucci D, Luppi G, Schena E, Beomonte Zobel B, et al. Percutaneous low-dose CT-guided lung biopsy with an augmented reality navigation system: validation of the technique on 496 suspected lesions. Clin Imaging. 2018;49:101-105.

5. Wang W, Yu L, Wang Y, Zhang Q, Chi C, Zhan P, et al. Radial EBUS versus CT-guided needle biopsy for evaluation of solitary pulmonary nodules. Oncotarget. 2018;9:15122-15131.

6. Schreiber G, McCrory DC. Performance characteristics of different modalities for diagnosis of suspected lung cancer: summary of published evidence. Chest. 2003;123(1 suppl):115S$128 \mathrm{~S}$.

7. Golse N, Ducerf C, Rode A, Gouillat C, Baulieux J, Mabrut JY. Transthoracic approach for liver tumors. J Visc Surg. 2012;149:e11-e22.

8. Thanos L, Zormpala A, Papaioannou G, Malagari K, Brountzos
E, Kelekis D. Safety and efficacy of percutaneous CT-guided liver biopsy using an 18-gauge automated needle. Eur J Intern Med. 2005;16:571-574.

9. Sconfienza LM, Mauri G, Grossi F, Truini M, Serafini G, Sardanelli F, et al. Pleural and peripheral lung lesions: comparison of US- and CT-guided biopsy. Radiology. 2013;266:930-935.

10. Ganott MA, Zuley ML, Abrams GS, Lu AH, Kelly AE, Sumkin JH, et al. Ultrasound Guided Core Biopsy versus Fine Needle Aspiration for Evaluation of Axillary Lymphadenopathy in Patients with Breast Cancer. ISRN Oncol. 2014 Feb 4;2014:703160. doi: 10.1155/2014/703160.

11. Yao X, Gomes MM, Tsao MS, Allen CJ, Geddie W, Sekhon H. Fine-needle aspiration biopsy versus core-needle biopsy in diagnosing lung cancer: a systematic review. Curr Oncol. 2012; 19:e16-e27.

12. Chang YY, Chen CK, Yeh YC, Wu MH. Diagnostic feasibility and safety of CT-guided core biopsy for lung nodules less than or equal to $8 \mathrm{~mm}$ : A single-institution experience. Eur Radiol. 2018;28:796-806.

13. Stattaus J, Kuehl H, Ladd S, Schroeder T, Antoch G, Baba HA, et al. CT-guided biopsy of small liver lesions: visibility, artifacts, and corresponding diagnostic accuracy. Cardiovasc Intervent Radiol. 2007;30:928-935.

14. Stattaus J, Kalkmann J, Kuehl H, Metz KA, Nowrousian MR, Forsting M, et al. Diagnostic yield of computed tomographyguided coaxial core biopsy of undetermined masses in the free retroperitoneal space: single-center experience. Cardiovasc Intervent Radiol. 2008;31:919-925.

15. Hsu MY, Pan KT, Chen CM, Lui KW, Chu SY, Lin YY, et al. CT-guided percutaneous core-needle biopsy of pancreatic masses: comparison of the standard mesenteric/retroperitoneal versus the trans-organ approaches. Clin Radiol. 2016;71:507-512.

16. Lin CY, Ou MC, Liu YS, Chuang MT, Shan YS, Tsai HM, et al. A CT-guided fat transversing coaxial biopsy technique for pancreatic lesion biopsy that avoids major organs and vessels. Saudi J Gastroenterol. 2017;23:341-347.

17. Odisio BC, Tam AL, Avritscher R, Gupta S, Wallace MJ. CT-guided adrenal biopsy: comparison of ipsilateral decubitus versus prone patient positioning for biopsy approach. Eur Radiol. 2012;22:1233-1239.

18. Iguchi T, Hiraki T, Matsui Y, Fujiwara H, Sakurai J, Masaoka $\mathrm{Y}$, et al. CT fluoroscopy-guided renal tumour cutting needle biopsy: retrospective evaluation of diagnostic yield, safety, and risk factors for diagnostic failure. Eur Radiol. 2018;28:283-290.

19. Davis IC, Heilbrun ME, Tangtiang K, Childs DD, Zagoria RJ. Computed tomography-guided renal tumor biopsies: tumor imaging features affecting sample adequacy. J Comput Assist Tomogr. 2013;37:171-175.

20. Monfardini L, Preda L, Aurilio G, Rizzo S, Bagnardi V, Renne $\mathrm{G}$, et al. CT-guided bone biopsy in cancer patients with suspected bone metastases: retrospective review of 308 procedures. Radiol Med. 2014;119:852-860.

21. Chang IJ, Ilaslan H, Sundaram M, Schils J, Subhas N. CTguided percutaneous biopsy of sclerotic bone lesions: diagnostic outcomes. Skeletal Radiol. 2018;47:661-669.

22. Heerink WJ, de Bock GH, de Jonge GJ, Groen HJ, Vliegenthart R, Oudkerk M. Complication rates of CT-guided transthoracic lung biopsy: meta-analysis. Eur Radiol. 2017;27:138-148.

23. Karcaaltincaba M, Akhan O. Radiologic imaging and percutaneous treatment of pelvic lymphocele. Eur J Radiol. 2005;55:340-354. 